\title{
Microstructural and Material Properties of Individual Zirconium Hydride Domains Formed Under Simulated Conditions
}

\author{
Shawn Riechers ${ }^{1}$, Brad Johnson ${ }^{2}$ \\ 1. Geochemistry Division, Pacific Northwest National Laboratory, Richland, Washington, USA. \\ 2. Nuclear Sciences Division, Pacific Northwest National Laboratory, Richland, Washington, USA
}

Zircaloy-4 is used as a fuel-element cladding in nuclear-power generation as well as a getter material for tritium production. The formation of hydride phases in Zircaloy-4 components alters material properties at the molecular scale leading to crack nucleation, embrittlement, and possible failure. The properties of individual micron sized domains and their effect on bulk properties is not well understood. Recent work with simulated Zircaloy-4 getter tube hydriding has shown that the $\mathrm{ZrH}_{\mathrm{x}}$ phases are present and their distribution is highly sensitive to seemingly minor differences in hydrogen loading conditions. In order to understand these hydriding behaviors and their implications for bulk material properties it is important to probe the material properties of individual domains. While bulk physical properties such as hardness [1] and magnetic susceptibility [2] have been measured and scanning electron microscopy (SEM) characterization using energy-dispersive $\mathrm{x}$-ray spectroscopy (EDX) and electron backscatter diffraction (EBSD) analysis has provided insight into the distribution and identity of individual Zircoloy-4 domain phases [3], little is known about the physical properties of individual micron scale domains and therefore how the resulting structures may affect Zircaloy-4 component properties and performance. By utilizing atomic force microscopy (AFM) we demonstrate it is possible to probe domain structures and physical properties on the nanometer scale to measure the fundamental material properties of individual hydride domains. This information can then be combined with correlative SEM to link material properties of individual domains to their chemical composition, structure, and $\mathrm{ZrH}_{\mathrm{x}}$ phase.

Hydriding Zicaloy-4 getter tubes under simulated reactor conditions at $500^{\circ \mathrm{C}}$ results in the formation of large domains at the boundary of $\mathrm{Zr}$ domains (Figure 1.A) while domains formed at $350^{\circ \mathrm{C}}$ are smaller, needle-like, and penetrate the $\mathrm{Zr}$ domains (Figure 1.B). The resulting hydride domains are identified by EBSD mapping as shown in Figure 1.C. In order to determine the material properties of individual domains formed under these conditions a multimodal AFM was used to map physical properties including hardness and the presence of magnetic domains. By mapping relative hardness individual domain boundaries are revealed that are not obvious from the topographical data as shown in Figure 2.A-B. Magnetic force microscopy, a two pass AFM imaging mode that measures topography and magnetic domains at the nanometer scale, revealed the presence of magnetic $\mathrm{ZrH}_{\mathrm{x}}$ phases (Figure 2.BC). This work showcases the ability to map physical properties previously only known at the bulk level to individual domains, revealing the effects of hydride formation conditions at the nanometer level. Additional AFM modes allow for detection of domain specific electrical and thermal properties. By utilizing correlated SEM and AFM imaging, the phase identity of individual domains can be matched with their physical properties. Once domain phases are known and AFM measurements, such as force maps are carefully calibrated they can be quantitatively compared to known values, such as the Young's modulus of bulk $\mathrm{ZrH}_{\mathrm{x}}$ phases, in order to investigate how local properties such as domain size, strain, and twinning affect the material properties of individual domains. By characterizing the resultant Zircoloy-4 hydride domain structures formed under varying conditions and measuring their material properties we can better understand, predict, and control hydride formation processes. 
[1] J Xu and S Shi, Journal of Nuclear Materials 327 (2004), 165.

[2] V Markin and V Savin, Inorganic Matter 19 (1983), 809.

[3] F Long et. al., Acta Materialia 129 (2017), 450.

[4] The authors acknowledge funding was provided as part of the Tritium Science Program within the National Nuclear Security Administration's Tritium Sustainment Program.
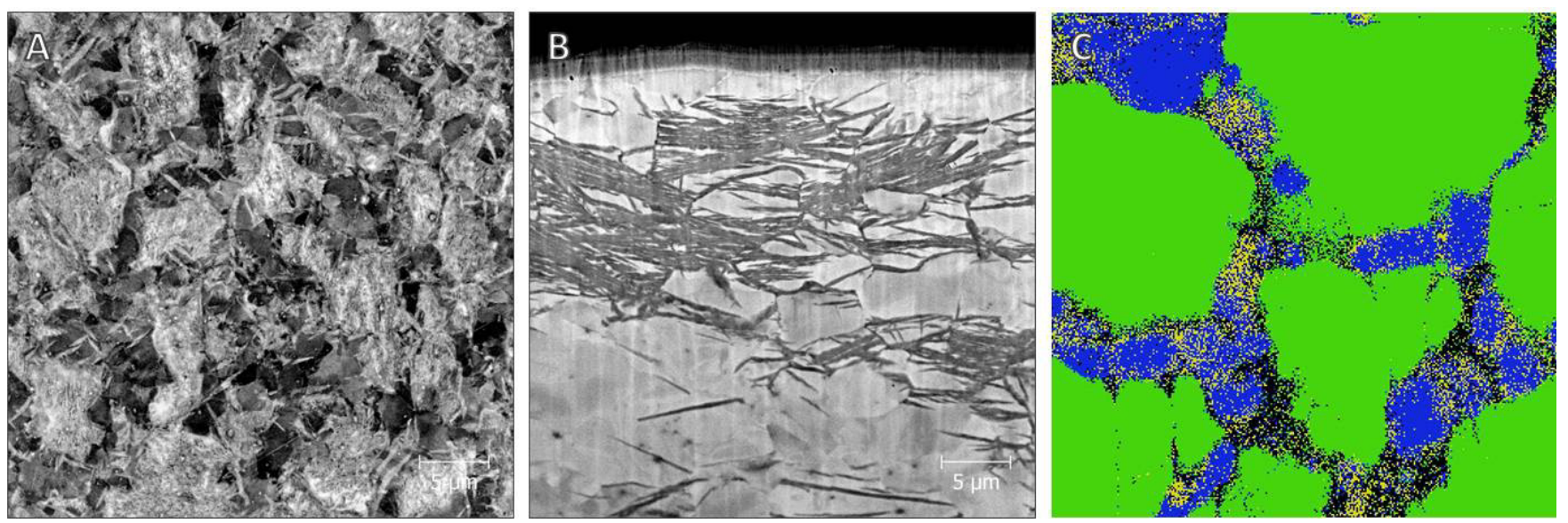

Figure 1. SEM micrographs of Zircaloy-4 tubing display the hydride domains formed at $500^{\circ} \mathrm{C}$ resulting in the formation of larger homogeneously distributed hydride domains (A), and at $350^{\circ \mathrm{C}}$ where smaller needle-like hydride domains form in bands that cut through the existing Zr domains (B). (C) SEM based EBSD provides the phase of Zircaloy-4 hydride domains, green $=$ alpha phase, blue $=$ delta phase, and yellow = gamma phase.
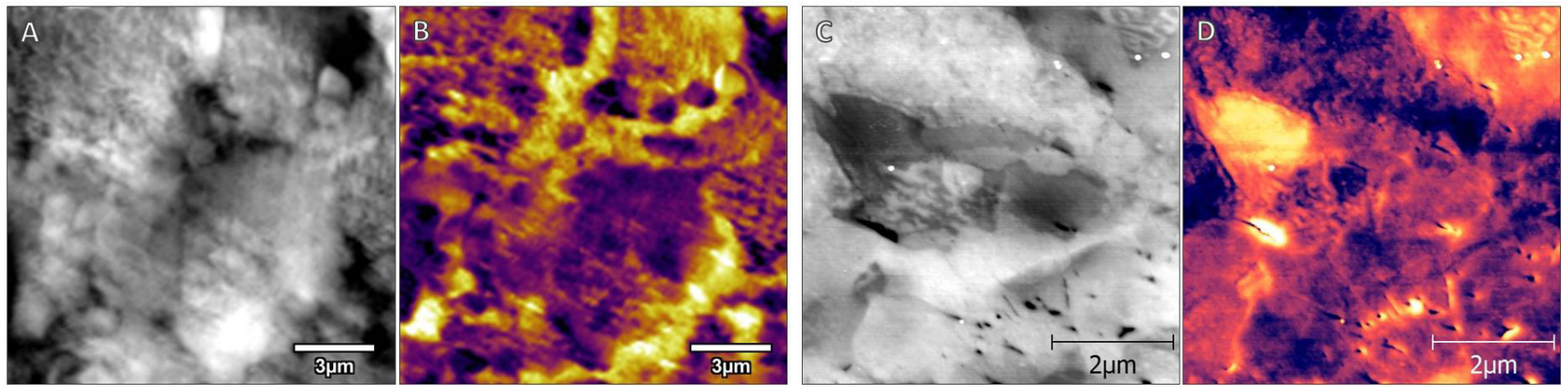

Figure 2. AFM based fast force mapping provides the topography of individual domains (A) correlated with their relative hardness (B). AFM based magnetic force microscopy was used to provide topography $(\mathrm{C})$ while revealing the presence of magnetically susceptible domains (D). 